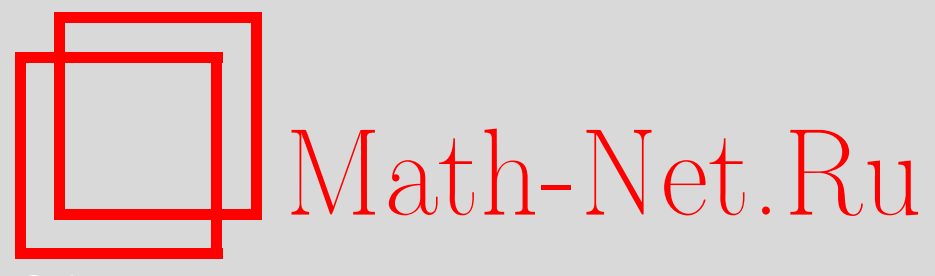

А. Заславский, В. Протасов, Как выйти из леса?, Квант, 2020, номер 9, 10-17

DOI: https://doi.org/10.4213/kvant20200902

Использование Общероссийского математического портала Math-Net.Ru подразумевает, что вы прочитали и согласны с пользовательским соглашением http://www.mathnet.ru/rus/agreement

Параметры загрузки:

IP: 54.157 .27 .8

26 апреля 2023 г., 16:02:03

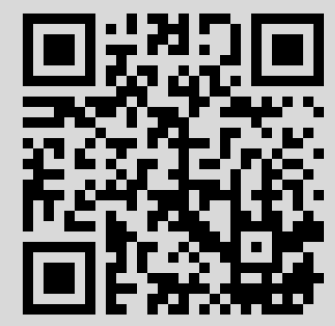




\section{Как выйти из леса?}

\section{А.ЗАСЛАВСКИЙ, В.ПРОТАСОВ}

Как нам быть и куда нам идти?

Как мы жили и правда ли жили?

Где свои, где ничьи, где чужие?

Что терять, где искать, как найти?

Л.Костюков

Днем, во втором часу,

Заблудилась принцесса в лесу.

Г.Сапгир. Принцесса и людоед

TrA АК ВЫЙТИ ИЗ ЛЕСА? ЧТО ЗА странный вопрос! Если есть навигатор или, на худой конец, карта и компас, то задача решается просто. Но если карты нет, а телефон разрядился? Тогда нужно идти все время прямо - когда-нибудь да выйдем. Однако эта стратегия опасна: если направление выбрано неудачно, то можно зайти очень далеко вглубь леса. Что же делать? Попробуем точно сформулировать задачу. Итак:

1. Нам неизвестно, в какой точке леса мы находимся, и неизвестны направления.

2. Что-то про лес мы все-таки знаем скажем, его площадь, форму и т.д.

3. Мы можем идти по любому заранее выбранному маршруту. Например, пройти точно по кругу с заданным центром и радиусом 10 км или, скажем, пройти 5 км в данном направлении, затем повернуть на $60^{\circ}$ и пройти 3 км и т.д.

Вопрос: как нужно идти, чтобы, пройдя по нашему маршруту, обязательно выйти из леса, независимо от начального положения и начального направления движения? Какова длина самого короткого маршрута, гарантирующего выход? Ответ, конечно, зависит от того, что мы знаем про лес.

Задача 1. Каков кратчайший маршрут, обеспечиваюший выход из леса, если мы

DOI: https://doi.org/10.4213/kvant20200902 знаем, что диаметр леса не превосходит $10 \kappa м$ ?

Диаметром фигуры называется расстояние между самыми далекими друг от друга точками этой фигуры. Таким образом, диаметр есть у любой ограниченной фигуры, а не только у круга. ${ }^{1}$ Значит, нам известно, что лес не очень большой: расстояние между любыми двумя его точками не превосходит 10 км.

Решение. Проходим 10 км по прямой. Всё, мы обязательно выйдем из леса либо к концу пути, либо еще раньше. Иначе лес будет содержать внутри себя отрезок длиной 10 км, а значит, диаметр леса будет больше 10 км.

И что, так просто? Идем по прямой наугад - и вся «стратегия»? Да. Более того, этот путь оптимальный. Это значит, что никакой путь длиной меньше 10 км не может гарантировать выход из леса. В самом деле, пройдем по любому пути длиной меньше 10 км. Обозначим его длину через $d$. А теперь накроем этот путь очень узкой полоской. Если $A$ и $B$ - любая пара точек на нашем пути, то длина отрезка $A B$ не превосходит длины участка пути от $A$ до $B$ (поскольку кратчайший путь между двумя точками проходит по прямой), а значит, $A B \leq d$. Итак, диаметр нашего пути, если его рассматривать как плоскую фигуру, не превосходит $d$. Поскольку $d<10$ км, если полоска достаточно узкая, ее диаметр также будет меньше 10 км. А теперь представим, что лес - это наша полоска. Диаметр леса меньше 10 км, но мы так из него и не вышли (ведь полоска содержит внутри себя весь наш путь).

Конечно, это была простая ситуация. А вот посложнее.

1 Все фигуры считаем замкнутыми, т.е. содержащими свою границу. 


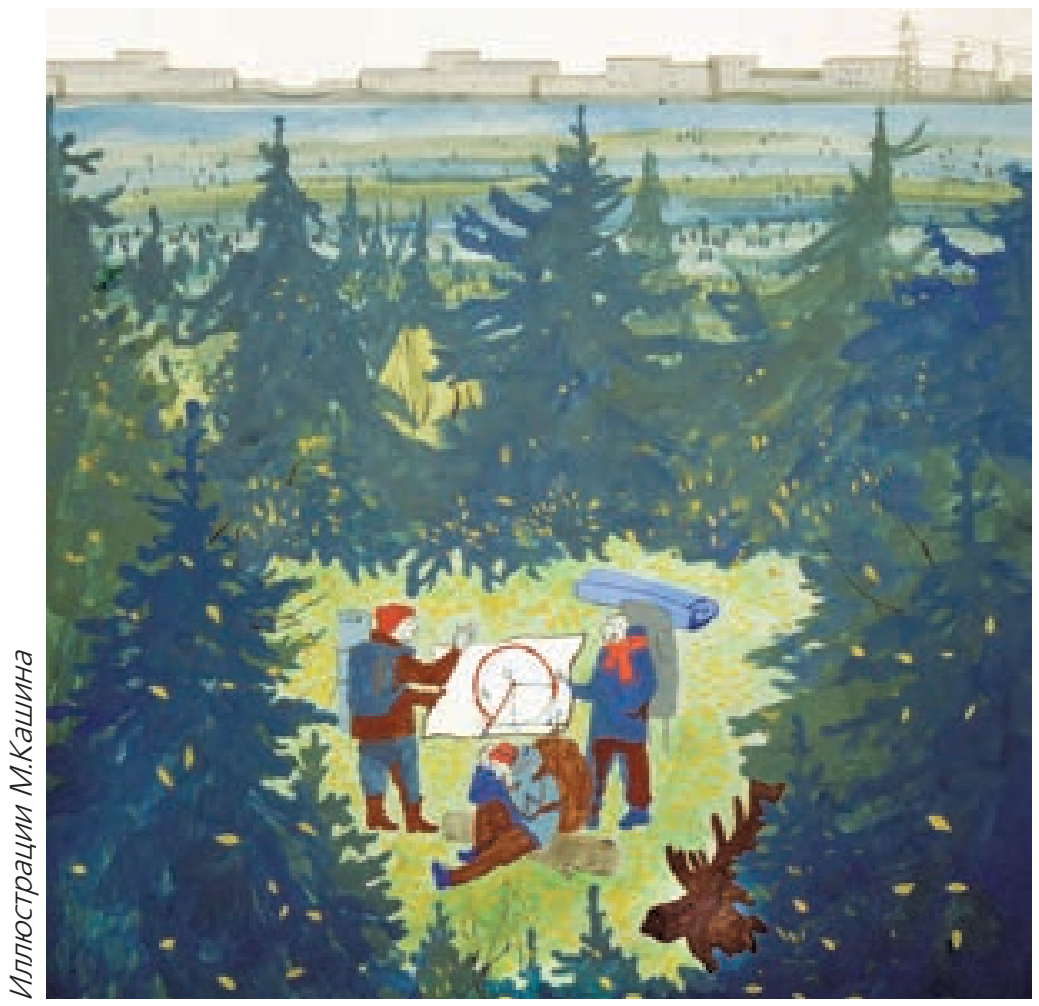

зательство такое же. Но при этом мы пройдем больше - сторона квадрата площади 16 км² $^{2}$ равна 4 км, поэтому длина пути равна 16 км. Круг в данной задаче является оптимальной фигурой. Для доказательства нам понадобится следующий факт.

Факт. Среди всех кривых заданной длины наибольшую площадь ограничивает окружность.

Факт этот известен всем. Даже маленький ребенок в магазине игрушек восклицает «Это всё мое, мое!», складывая руки в круг, демонстрируя тем самым, что именно круг вмещает больше всего. Данное свойство круга называется изопериметрическим (в переводе с греческого - «рав-

Задача 2. Площадь леса $16 \kappa м^{2}$, и в нем нет полян. Каков кратчайший путь выхода из леса?

Нет полян - значит, лес сплошной, без «дырок».

Решение. Идем по границе круга площадью 16 км $^{2}$ и обязательно выходим из леса. Иначе говоря, проходим всю окружность радиусом $\sqrt{\frac{16}{\pi}}=\frac{4}{\sqrt{\pi}}=2,256 \ldots$ км. Направление начального движения (т.е. положение центра окружности) не важно. При этом длина пути составит $2 \pi \frac{4}{\sqrt{\pi}}=$ $=8 \sqrt{\pi}=14,179 \ldots$ км.

То, что мы выйдем из леса, понять легко. Если лес содержит внутри себя весь путь окружность, то он содержит и весь круг (полян нет!), а значит, его площадь должна быть больше 16 км² $^{2}$

А что будет, если мы идем не по кругу, a, скажем, по квадрату той же площади? Выйдем ли мы из леса? Конечно. И доканопериметрическим»). Итак, из всех фигур с равными периметрами именно круг имеет бо́льшую площадь. Несмотря на геометрическую очевидность, это довольно сложно обосновать. Известно изопериметрическое свойство с глубокой античности, оно имеет массу объяснений, как геометрических, так и физических, и даже философских. Но строго доказано оно было лишь в XIX веке (см. $[1,2])$.

Докажем оптимальность кругового маршрута. Если наш маршрут незамкнутый, то его можно поместить в тонкую полоску маленькой площади. Если лес занимает эту полоску, то он, даже при длинном маршруте, может иметь сколь угодно маленькую площадь. А маршрут из леса не выведет. Таким образом, мы смотрим только на замкнутые маршруты. Из изопериметрического свойства круга следует, что замкнутый маршрут длины $8 \sqrt{\pi}$ км (или меньшей) не может ограничивать площадь большую, чем окружность той же длины, т.е. площадь 16 км² $^{2}$ Если лес является 
фигурой, ограниченной нашим маршрутом, то его площадь не превосходит 16 км², и мы проходим $8 \sqrt{\pi}$ км, не выходя из леса (хотя, возможно, часть пути идем по краю, не замечая этого). Противоречие. Оптимальность круга доказана.

Итак, для леса без полян круговой маршрут оптимален. А что будет при дополнительном предположении, что лес выпуклый?

Задача 3. Плошадь леса $16 \kappa м^{2}$, и лес выпуклый. Каков теперь кратчайший путь выхода из леса?

Фигура называется выпуклой, если вместе с любыми двумя своими точками она целиком содержит отрезок, соединяющий эти точки. Окружность невыпукла, а круг выпуклый. Выпуклые фигуры изучает красивая и содержательная область математики - выпуклая геометрия. Ее результаты лежат в основе выпуклой оптимизации, имеющий широкие применения в современном мире.

Решение. Выпуклый лес не имеет полян. Иначе можно взять точки на разных концах поляны и соединить их отрезком. Отрезок пройдет через поляну, что противоречит выпуклости леса. И раз полян нет, круговой маршрут из задачи 2 также годится для выпуклого леса. Но будет ли он оптимальным?

Предположим, мы прошли $a$ км и пришли из точки $A$ в точку $B$. Если мы так и не вышли из леса, то лес целиком содержит наш маршрут. А поскольку лес выпуклый, он содержит и отрезок $A B$. Итак, лес содержит фигуру, ограниченную замкнутой кривой: наш маршрут + отрезок $A B$. Какова максимальная площадь такой фигуры? Обозначим эту площадь через $S$. Изопериметрическое свойство применять нельзя, поскольку длина кривой неизвестна - мы не знаем длину $A B$. Поэтому поступим так: отразим путь симметрично относительно прямой $A B$. Наш путь вместе с отраженным образуют замкнутую кривую длины $2 a$, ограничивающую фигуру площади $2 S$. К этой фигуре применяем изопериметрическое свойство и приходим к выводу, что ее площадь максимальна, когда она ограничена окружностью длины 2a. Следовательно, оптимальный путь полуокружность длины $a$, т.е. радиусом $\frac{a}{\pi}$. Площадь ограничиваемого полукруга должна быть равна 16 км² $^{2}$ откуда

$$
16=\frac{1}{2} \pi R^{2}=\frac{1}{2} \pi\left(\frac{a}{\pi}\right)^{2}=\frac{a^{2}}{2 \pi} .
$$

Следовательно, $a=4 \sqrt{2 \pi}$ и $R=\frac{4 \sqrt{2}}{\sqrt{\pi}}$.

Итак, мы должны идти по полуокружности радиусом $\frac{4 \sqrt{2}}{\sqrt{\pi}} \approx 3,191 \ldots$ км. Это оптимальный путь. Его длина составляет $a=4 \sqrt{2 \pi} \approx 10,026 \ldots$ км. Для сравнения: круговой маршрут имеет длину $8 \sqrt{\pi} \approx$ $\approx 14,179$.. км.

Признаемся, что элегантный трюк с симметрией придумали не мы (знающий читатель, конечно, заметил это). Так решается задача Дидоны об огораживании максимального участка земли от прямого берега моря (см. [1,2]).

Подведем некоторые итоги. Человек находится внутри леса. Он знает форму леса, но не имеет (или имеет неполную) информации о своем местоположении. Требуется найти траекторию наименьшей длины, гарантирующую выход из леса при любой начальной точке и любом направлении движения. По-видимому, такие задачи впервые были рассмотрены в 1954 году выдающимся американским математиком Р.Беллманом ${ }^{2}$ в книге [4] и получили название «задачи о выходе из леса». А в 1961 году замечательный советский геометр В.А.Залгаллер ${ }^{3}$ в своей статье [3] приводит решения нескольких подобных

2 Ричард Эрнест Беллман (1920-1984) американский математик, один из основателей теории оптимального управления и динамического программирования.

${ }^{3}$ Виктор Абрамович Залгаллер (1920-2020) советский математик, известный работами по многогранникам, дифференциальной геометрии, выпуклой оптимизации. Много сделал для развития математического образования. Из его кружка вышли несколько выдающихся математиков. 
задач. Одну из них мы рассмотрим сейчас. На этот раз лес бесконечен. Для простоты мы будем пользоваться безразмерными единицами длины.

Задача 4. Лес имеет форму бесконечной полосы ширины 1. Каков кратчайший путь выхода из такого леса?

Решение. Будем следовать статье Залгаллера. Нам понадобятся два вспомогательных утверждения. Кривую без самопересечений будем называть дугой. Ясно, что кратчайший путь надо искать именно среди дуг. Дугу будем называть выпуклой, если она вместе с отрезком, соединяющим ее концы, ограничивает выпуклую фигуру.

Лемма 1. Существует путь минимальной длины. Он является выпуклой дугой.

А что тут доказывать? Ну конечно существует кратчайший путь, это же очевидно! Увы, в некоторых задачах на поиск минимума/максимума оптимальное решение не достигается. Более того, именно существование решения порой труднее всего доказать. А не доказывать нельзя - иначе можно прийти к абсурдным выводам (см. несколько примеров в [2]). Данная задача - не исключение. Поэтому лемма 1 очень важна. Но доказывать ее здесь мы все-таки не будем, а примем ее без доказательства и отошлем заинтересованного читателя к статье [3].

Выясним теперь, какими свойствами должна обладать кратчайшая кривая, назовем ее $L$. Нам понадобится понятие опорной прямой выпуклой фигуры. Это прямая, которая имеет с данной фигурой общие точки, но при этом вся фигура лежит по одну сторону от прямой. Например, опорные прямые круга - это касательные. А опорные прямые треугольника - это три прямые, содержащие его стороны, а также прямые, проходящие через его вершины и не имеющие других точек с треугольником.

Лемма 2. Кониы $A$, B кратчайшей кривой L не совпадают друг с другом, $и L$ расположена между перпендикулярами к отрезку $A B$ в его кониах.

Доказательство. Проведем перпендикулярные к прямой $A B$ опорные прямые к $L$, изображенной синим цветом (рис.1). Если точки $A^{\prime}, B^{\prime}$ не совпадают с $A$ и $B$ соответственно, то, заменив дуги $M A, N B$ отрезками $M A^{\prime}, N B^{\prime}$, мы получим кривую

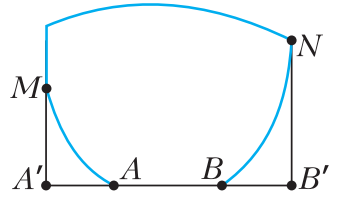
короче $L$, которая, очевидно, также не помещается в полосе.

Обозначим теперь длину отрезка $A B$ через $x$, а прямую $A B$ через $a$. Из леммы 2 следует, что $x \geq 1$, а $L$ лежит внутри полуполосы, ограниченной отрезком $A B$ и перпендикулярами $a, b$ к нему в его концах. Пусть $C C^{\prime}, D D^{\prime}-$ четверти дуг окружностей единичного радиуса с центрами $A$ и $B$ соответственно (рис. 2). Так как $L$ не

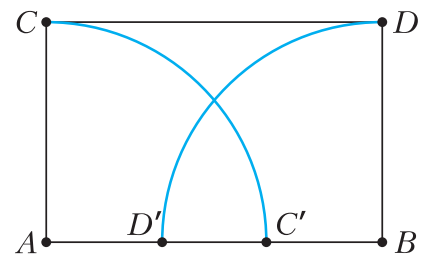

РИс. 2

помещается в единичной полосе, она должна достигать в какой-то точке отрезка $C D$. Кроме того, на пути от $A$ до $C D$ дуга $L$ не должна пересекать сектор $B D D^{\prime}$, а на пути от $C D$ до $B$ - сектор $A C C^{\prime}$. Будем для каждого фиксированного $x \geq 1$ искать кратчайшую из кривых, обладающих этими свойствами.

Пусть $M$ - точка, в которой искомая кривая достигает отрезка $C D$. Тогда ее участок $A M$ либо является отрезком, либо состоит из касательных, проведенных из точек $A, M$ к дуге $D D^{\prime}$, и дуги между точками касания (докажите это!). Аналогичное утверждение верно и для участка $M B$. Кроме того, для кратчайшей кривой $M$ должна совпадать с серединой отрезка $C D$ или с одним из его концов. Действительно, в противном случае можно провести прямую, параллельную $C D$ и достаточно близкую к ней, которая отсечет от кривой треугольник. Заменив этот треугольник равнобедренным с теми же основанием и высотой, мы уменьшим длину кривой. Теперь докажите самостоятельно, что при $M=C$ или $M=D$ кривая также не будет кратчайшей. 


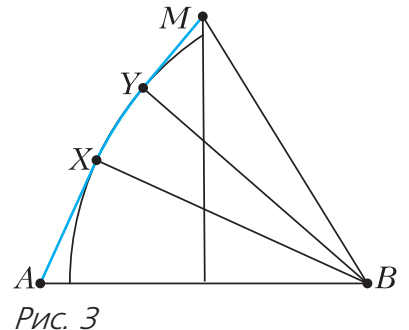

Таким образом, при любом $x$ кратчайшей будет симметричная кривая $L(x)$, состоящая из кратчайших путей, соединяющих точки $A$ и $B$ с серединой $M$ отрезка $C D$. Найдем теперь, при каком $x$ такая кривая имеет наименьшую длину. При $x \geq 2 / \sqrt{3}$ кратчайшие пути $A M, M B$ являются отрезками, длины которых возрастают с ростом $x$, поэтому надо найти минимум длины $L(x)$ при $1 \leq x \leq 2 / \sqrt{3}$.

Пусть $A X, M Y$ - касательные из точек $A$, $M$ к дуге $D D^{\prime}$ (рис. 3), $\alpha=\angle A B X, \beta=$ $=\angle M B Y=\pi / 2-\angle A B M$. Тогда $\cos \alpha=1 / x$, $\operatorname{tg} \beta=x / 2, \angle X B Y=\pi / 2-2 \beta$ и, следовательно, длина кривой $A X Y M$ равна

$$
\begin{aligned}
\sqrt{x^{2}-1}+x / 2+ & \pi / 2- \\
& -\arccos (1 / x)-2 \operatorname{arctg}(x / 2) .
\end{aligned}
$$

Для того чтобы найти минимум этой функции, исследуем ее с помощью производной. Производная обращается в ноль в единственной точке $x \approx 1,0436$, при этом длина кривой $L(x)$ примерно равна $2,278 \ldots$

Заметим, что найденная кривая является оптимальной также для леса, имеющего форму прямоугольника с достаточно большим отношением сторон. Но для прямоугольников, близких к квадрату, решение авторам неизвестно.

Мы разобрали четыре задачи о выходе из леса. В них человек знает некоторые геометрические параметры леса, но не имеет представления о своем местоположении. Если же человеку что-то известно о том, где он находится (скажем, на каком расстоянии от границы леса), то это уже другой тип задач. Как ни странно, порой более сложный.

Задача 5. Лес имеет форму полуплоскости, а человек находится на расстоянии
1 от ее гранищь. Каков кратчайший путь выхода из леса в этом случае?

В решении мы используем еще одно понятие из выпуклой геометрии. Выпуклая оболочка произвольного множества точек $A-$ это наименьшее выпуклое множество, содержащее $A$. Наименьшее - значит, оно лежит в любом выпуклом множестве, содержащем $A$. Выпуклая оболочка есть у каждого множества. Например, выпуклая оболочка двух точек - отрезок, соединяющий данные точки. Выпуклая оболочка трех точек, не лежащих на одной прямой, - треугольник с вершинами в данных точках. Выпуклая оболочка окружности и точки вне ее ограничена двумя касательными из точки к окружности и дугой, соединяющей точки касания. На рисунке 4 изображена выпуклая оболочка окружности и не

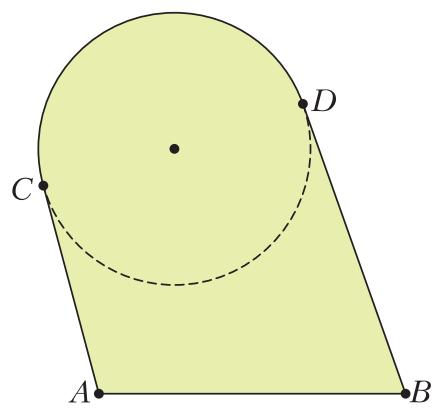

Pис. 4

пересекающего ее отрезка $A B$, она ограничена отрезком, касательными $A C$ и $B D$ к окружности и дугой $C D$. Выпуклую оболочку фигуры можно строить по такому правилу: выпуклая оболочка ограничена всеми опорными прямыми к данной фигуре.

Решение. Пусть $K$ - единичный круг с центром в точке $O$, где находимся мы. Если выпуклая оболочка пути не содержит круг $K$, то к нему можно провести касательную

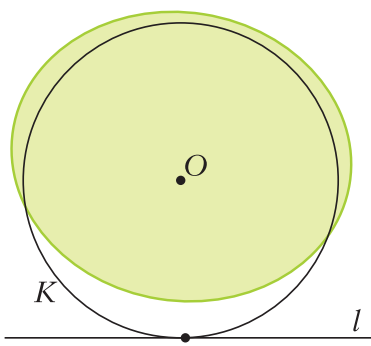

Pис. 5 $l$, не пересекающую нашего пути (рис. 5). Очевидно, что в этом случае путь не гарантирует выход из леса. С другой стороны, если $K$ содержится в выпуклой оболочке, 
то любая касательная к этому кругу пересекает наш путь, и выход гарантирован. Поэтому оптимальный путь должен состоять из отрезка $O X$, ведущего в некоторую точку $X$, лежащую вне или на границе $K$ и дуги $X Y$, выпуклая оболочка которой содержит $K$.

Лемма 3. Для кратчайшей кривой отрезок XY касается $K$, акривая XY состоит из касательных $X A, Y B \kappa K и$ ограничиваюшей его дуги $A B$.

Доказательство. Для любой кривой $X Y$, выпуклая оболочка которой, назовем ее $F$, содержит круг $K$, можно построить кривую, удовлетворяющую условию леммы, выпуклая оболочка которой также содержит $K$ и содержится в $F$. (Можно представить себе, что в точках $X, Y$ вбиты гвозди, к которым прикреплена охватывающая $K$ резинка. Очевидно, что резинка примет форму, указанную в условии леммы.)

Таким образом, задача сводится к следующей. Пусть прямая $l$ касается $K$ в некоторой точке $C$. Выберем точки $A, B$ на границе $K$ и проведем в них касательные, пересекающие $l$ в точках $X, Y$ соответственно. Требуется минимизировать длину кривой $O X A B Y$ (рис. 6).

Обозначив углы $\angle A O C=\alpha, \angle B O C=\beta$, получим, что длина пути равна $1 / \cos \alpha+$ $+\operatorname{tg} \alpha+\operatorname{tg} \frac{\beta}{2}+2 \pi-2 \alpha-\beta$. Минимум этой функции достигается при $\alpha=\pi / 6, \beta=\pi / 2$ и равен $6,395 \ldots$

Итак, мы нашли путь для выхода из леса и доказали его оптимальность. Это путь, изображенный на рисунке 6.

Интересно, что в книге Беллмана и в статье Залгаллера приведен более длин-

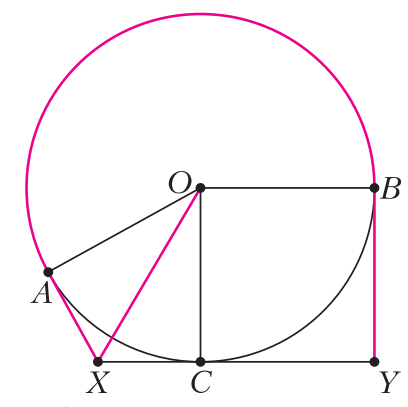

Рис. 6 ный путь. А оптимальное решение, которое мы сейчас нашли, было впервые получено американским математиком Джоном Исбеллом в 1957 году в его статье [5]. Беллман назвал это решение гениальным («The author solves this problem in an ingenious fashion ...»). Интересно также, что Залгаллер в своей статье [3] ссылается на решение Исбелла, но воспроизводит его неверно, получая тем самым неоптимальный путь. Мы подозреваем, что американский «Флотский журнал», в котором была напечатана статья Исбелла, был в то время в нашей стране недоступен, и Виктор Абрамович вынужден был пользоваться данными «Реферативного журнала», в котором публикуются краткие описания научных статей. А эти описания могли быть неточны.

1954 год, 1957 год, 1961 год... Для молодого читателя это - доисторические даты. А есть ли интерес к этой теме сейчас? Да! Например, следующая задача прозвучала совсем недавно: в 2019 году она предлагалась на Геометрической олимпиаде имени И.Ф.Шарыгина. И хотя в ней речь идет о причаливании корабля к берегу, она относится к тому же типу задач о выходе из леса.

Задача 6. Корабль в тумане пытается пристать к берегу. Экипаж не знает, в какой стороне находится берег, но видит маяк, находящийся на маленьком острове в 10 км от берега, и понимает, что расстояние от корабля до маяка не превышает 10 км (точное расстояние до маяка неизвестно). Маяк окружен рифами, поэтому приближаться к нему нельзя. Может ли корабль достичь берега, пропльв не больше 75 км? (Береговая линия - прямая, траектория до начала движения вычерчивается на дисплее компьютера, после чего автопилот ведет корабль по ней.)

Если бы не было условия, запрещающего приближаться к маяку, то, подплыв к нему вплотную, мы свели бы задачу к задаче 5 и получили бы траекторию с 


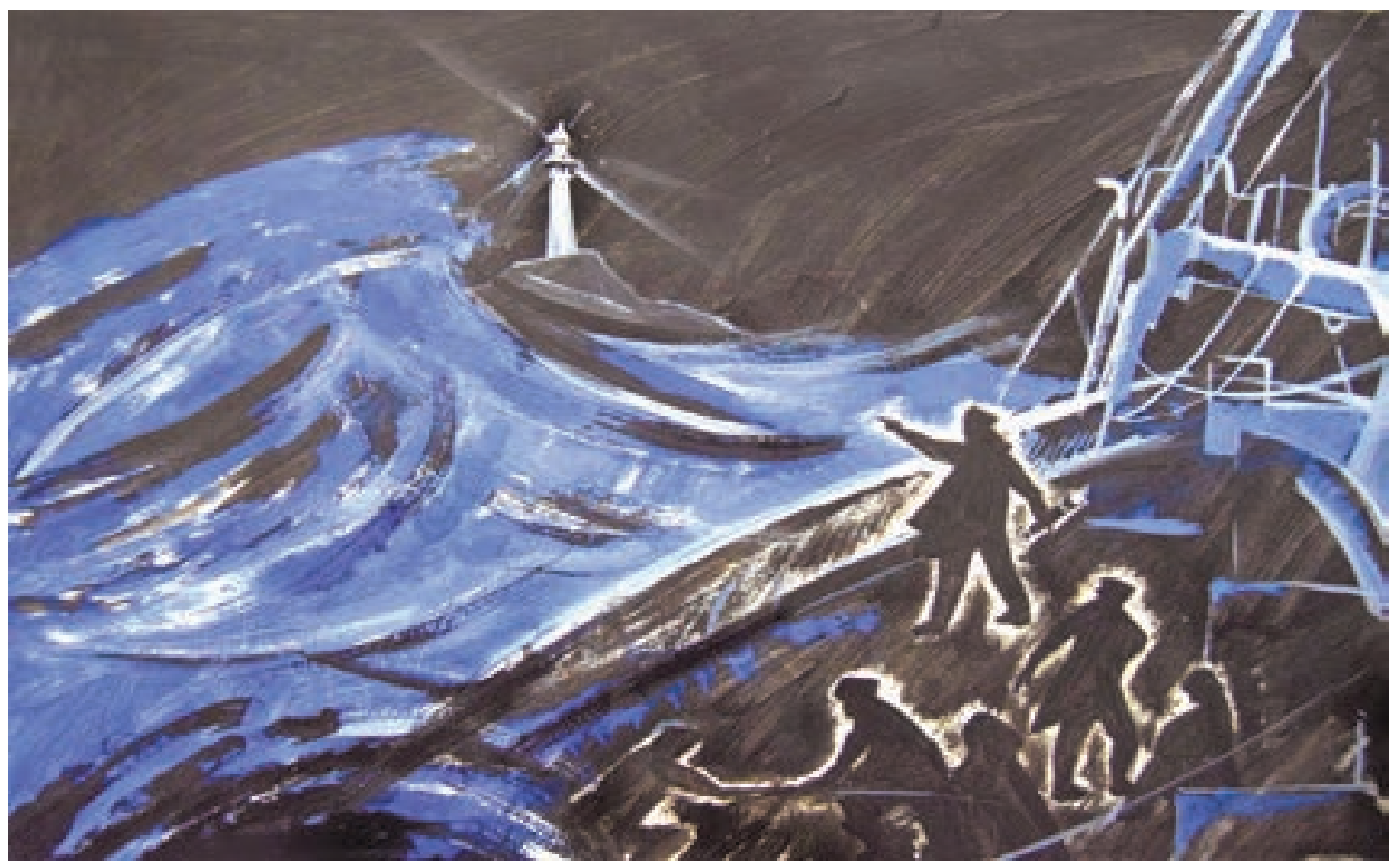

длиной, меньшей 74 км. Впрочем, решением задачи 5 все равно можно воспользоваться. Пусть корабль находится в точке $K$, маяк - в точке $M$, а $K^{\prime}$ - точка на луче $K M$ такая, что $K K^{\prime}=10$ км. Чтобы корабль причалил к берегу, выпуклая оболочка его траектории должна содержать круг с центром $M$ и радиусом $K K^{\prime}$, но, поскольку положение точки $M$ на отрезке $K K^{\prime}$ неизвестно, выпуклая оболочка должна содержать объединение всех таких кру-

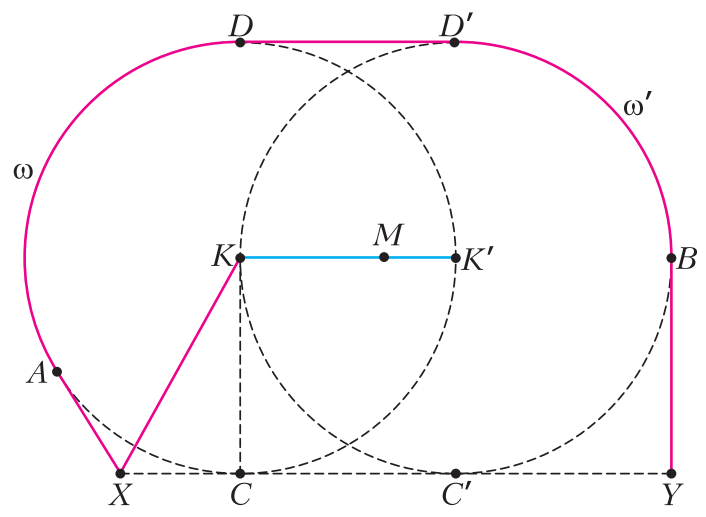

Pnc. 7 гов с центрами на $K K^{\prime}$. Ясно, что это условие будет и достаточным.

Пусть $\omega, \omega^{\prime}-$ окружности с центрами $K$, $K^{\prime}$ соответственно и радиусами, равными $K K^{\prime} ; C C^{\prime}$ и $D D^{\prime}$ - общие касательные к этим окружностям; $X$ - точка на прямой $C C^{\prime}$ такая, что $\angle X K C=30^{\circ} ; X A-$ касательная к $\omega ; B$ - середина дуги $C^{\prime} D^{\prime}$, лежащей вне $\omega ; Y$ - проекция $B$ на $C C^{\prime}$ (рис. 7). Тогда путь $K X A D D^{\prime} B Y$ удовлетворяет условию, а его длина равна $10(\sqrt{3}+2 \pi / 3+1+\pi / 2+1)$ км $<74$ км.

Повторяя рассуждение из решения задачи 5, мы приходим к выводу, что указанный путь является кратчайшим среди всех, для которых прямая $X Y$ совпадает с $C C^{\prime}$. Интуитивно кажется очевидным, что кратчайший путь должен удовлетворять этому условию, но доказательство авторам неизвестно. Возможно, читатель сможет его найти.

Задачи о выходе из леса имеют непосредственные практические приложения. И их не следует понимать буквально: к «выходу из леса» они часто не имеют никакого 
отношения. Это и построение оптимальных стратегий, и теория игр, и динамическое программирование. Достаточно упомянуть, что Дж.Исбелл выбрал для своей статьи именно «Флотский журнал», который посвящен прикладным инженерным и экономическим проблемам морских перевозок. Много прикладных аспектов «выхода из леса» можно найти в книге [6]. Есть ли в этой науке нерешенные задачи? Конечно! Они связаны как с различными формами леса, так и с иными постановками самой проблемы. Например, на практике нас интересует не столько путь, обеспечивающий выход всегда, сколько - обеспечивающий выход с высокой вероятностью. А такой путь может быть значительно короче! Иногда задача ставится о минимизации среднего времени выхода, т.е. математического ожидания.

В заключение предлагаем вам попробовать свои силы в решении еще нескольких задач.

7. Лес имеет форму единичного круга. Докажите, что кратчайшей траекторией выхода из леса является отрезок длины 2.

8. Лес имеет форму круга радиусом 10 , а человек находится в лесу на расстоянии 1 от его границы.

а) Обеспечивает ли путь из задачи 5 выход из леса?

б) Существует ли более короткий путь?

9. Лес имеет форму прямого угла, а человек находится на расстоянии 1 от его вершины. Можно ли выйти из леса, пройдя расстояние, не большее 3 ?

10. Лес имеет форму квадрата. Можно ли выйти из него, пройдя расстояние, меньшее диагонали квадрата?

11. Лес имеет форму правильного треугольника. Можно ли выйти из него, пройдя расстояние, меньшее стороны треугольника?

В последних четырех задачах авторы не знают, какой путь является кратчайшим.

\section{Литература}

1. В.М.Тихомиров. Рассказы о максимумах и минимумах. - Библиотечка «Квант», вып. 56. М.: Наука, 1986.

2. В.Ю.Протасов. Максимумы и минимумы в геометрии. - Библиотека «Математическое просвещение», вып. 31. - М.: МЦНМО, 2005.

3. В.А.Залгаллер. Как выйти из леса? (Об одной задаче Беллмана). - «Математическое просвещение», сер. 2 (1961), № 6, с.191-195.

4. R.Bellman. Dynamic Programming. - Princeton University Press, 1957.

Русский перевод: Р.Беллман. Динамическое программирование. - М.: Издательство иностранной литературы, 1960.

5. J.R. Isbell. An optimal search pattern. - Naval Research Logistics Quart Ferly, 4 (1957), 357-359.

6. P.J.Nahin. Chases and Escapes: The Mathematics of Pursuit and Evasion. - Princeton University Press, 2007.

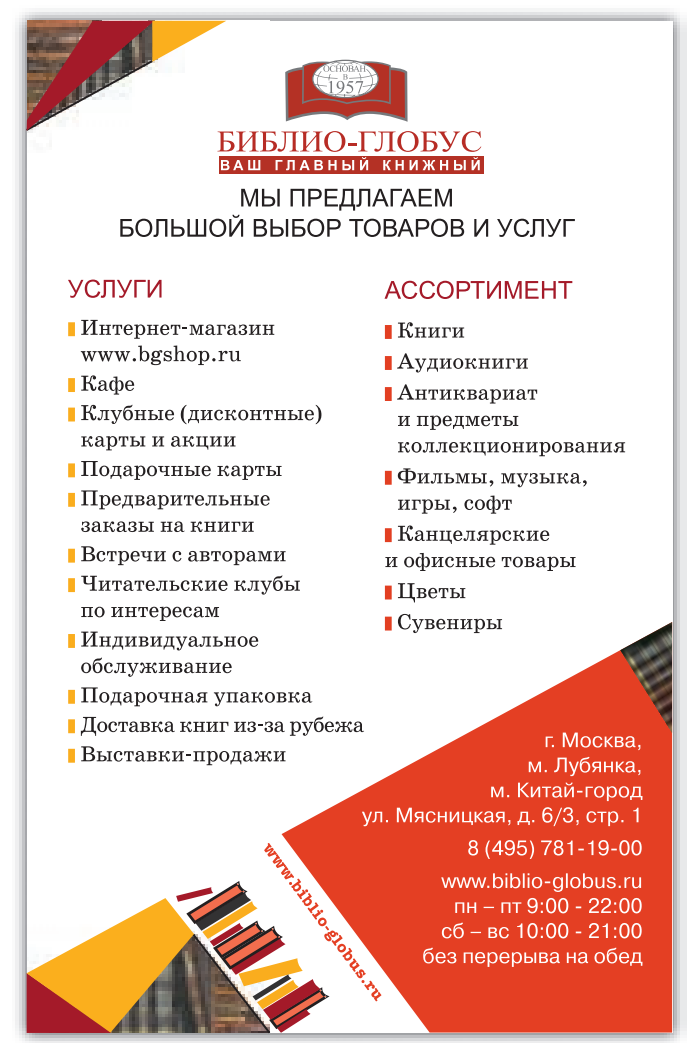

